\title{
Picometer mechanical displacement measurement using heterodyne interferometer with phase-locked loop
}

\author{
Masato Aketagawa ${ }^{1, *}$, and Thanh Dong Nguyen ${ }^{1}$ \\ ${ }^{1}$ Department of Mechanical Engineering, Nagaoka University of Technology, 940-2188 Kamitomioka, Nagaoka, Niigata, Japan
}

\begin{abstract}
In this paper, we show picometer-order mechnical displacmment measurements using a heterodyne interferometer with a phase-locked loop (PLL). A heterodyne light source for the interferometer is implemented with a frequency stabilized HeNe laser and two acousto-optic modulators. A real time phase measurement is performed by the PLL, whose software is programmed in a field-programmable gate array (FPGA). A stiff parallel spring stage combined with a high-voltage piezoelectric actuator is used to generate picometer-order mechanical motion. With the above implementations, mechanical displacement of 10 picometer or less can be measured.
\end{abstract}

\section{Introduction}

With the rapid progress of ultraprecision engineering and semiconductor manufacturing, precise metrology systems based on laser interferometers with resolution of 10 picometer order have become necessary for displacement/distance measurements [1]. In recent years, displacement-measuring heterodyne interferometers have become suitable for industrial applications [2], because they have traceability to the standard definition of the meter if frequencies of light sources are stable. In this paper, we show picometer-order mechnical displacmment measurements using a heterodyne interferometer with a phase-locked loop (PLL). A heterodyne light source for the interferometer is implemented with a frequency stabilized HeNe laser and two acousto-optic modulators (AOMs). A real time phase measurement is performed by the PLL, whose software is programmed in a fieldprogrammable gate array (FPGA). A stiff parallel spring stage combined with a high-voltage piezoelectric actuator is used to generate picometer-order mechanical motion.

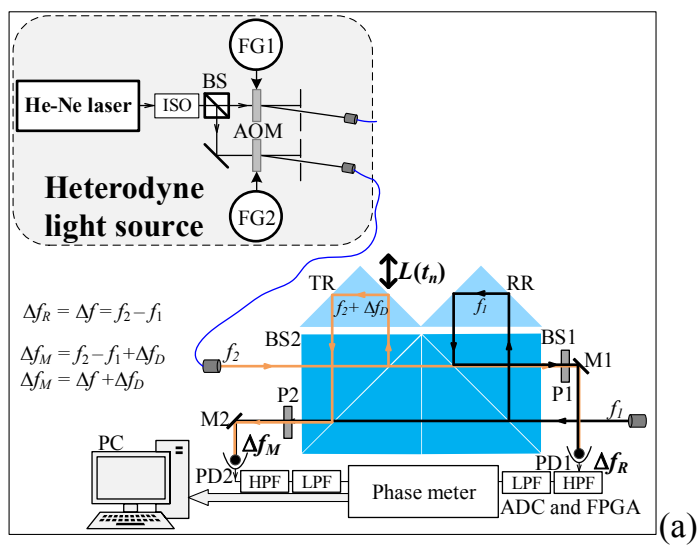

With the above implementations, mechanical displacement of 10 picometer or less can be measured.

\section{Implementation and Experiment}

Figure 1 shows a heterodyne interferometer with a heterodyne light source (= a HeNe laser + two AOMs). The heterodyne light source emits two bemas with difference frequencies $f_{1}$ and $f_{2}\left(f_{2}>f_{1}\right)$. The interferometer consists of two beam splitters (BSs) and two retro reflectors (target $\&$ refrence reflectors: TR \& RR). TR is moved by the parallel spring stage, whose resonant frequency is higher than $1 \mathrm{kHz}$, combined with the highvoltage piezoelectric actuator. The two beams enter to two BSs, TR, and RR. Then the mixed beams go to photo detectors (PD1 and PD2). At PD1, the reference beat signal $\left(\Delta f=f_{2}-f_{1}\right)$ is measured, at PD2, the measurement beat signal $\left(f_{2}-f_{1}+\Delta f_{\mathrm{D}}=\Delta f+\Delta f_{\mathrm{D}}\right.$, where $\Delta f_{\mathrm{D}}$ is Doppler frequency due to the movement of TR) is measured. The reference and measurement beat signals go to the phase

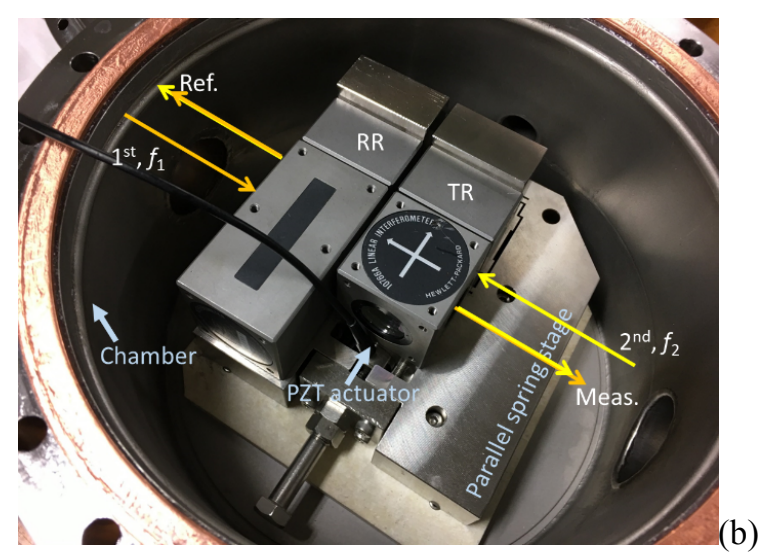

Figure 1. Schematic diagram (a) and photo (b) of the proposed heterodyne interferometer. FG: Function Generator, ISO: Isolator, BS: Beam splitter, TR\&RR: Target and reference retro reflectors, PD: Photo detector, P: Polarizer, LPF\&HPF: Low-pass \& highpass filters, ADC: Analogue to digital converter, M: Mirror, PZT: Piezo electric transducer.

\footnotetext{
* Corresponding author: masatoaa@,vos.nagaokaut.ac.jp
} 


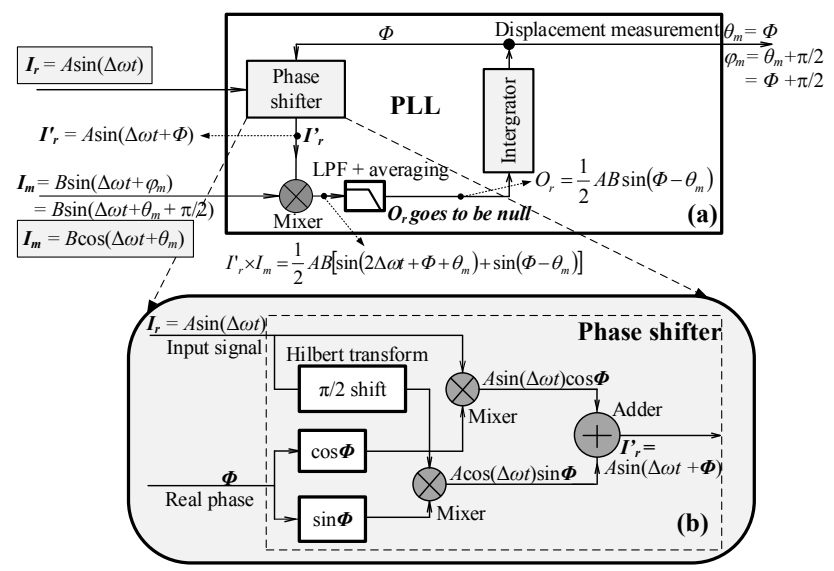

Figure 2. Schematic diagram of the phase meter using two sinusoidal heterodyne signals: (a) whole system and (b) phase shifter. PLL, phase-locked loop, LPF + averaging, low-pass filter combined with an averaging operation.
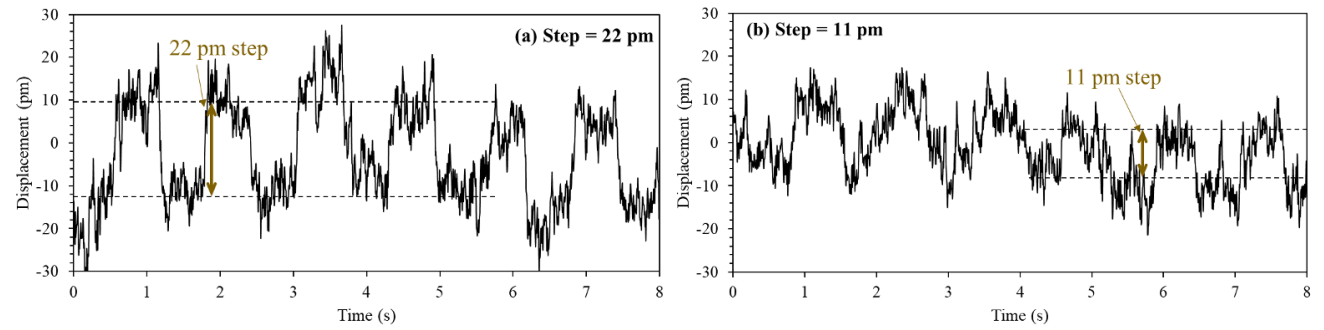

Figure 3. Step displacement measurement results obtained by the proposed system. (a) $22 \mathrm{pm}$ step (0.44 mrad) and (b) $11 \mathrm{pm}$ step $(0.22 \mathrm{mrad})$.

meter to determine the displacement of TR. Figure 2 shows the phase meter with PLL, whose softoware is programmed in the FPGA. The PLL consists of an active phase shifter with Hilbert transform, a mixer, a low-passfilter (LPF) with an averaging operation, and an integrator. The 120MHz/16bit analogue-to-digital converters (ADC) are used to digitize the reference and measurement beat signals. In the experiment, the beat frequency $\left(\Delta f=f_{2}-f_{1}\right)$ is $2.4 \mathrm{MHz}$. The input signals of the phase meter are the reference $I_{r}$ and measurement $I_{m}$ signals from PD1 and PD2 as shown in Figure 1. These signals $I_{r}$ and $I_{m}$ are

$I_{r}=A \sin 2 \pi \Delta f t=A \sin \Delta \omega t$

$I_{m}=B \sin \left(\Delta \omega t+\varphi_{m}(t)\right)=B \cos \left(\Delta \omega t+\theta_{m}(t)\right)(2)$,

where $A(B) \quad \Delta \omega$, and $\varphi_{m}(t) \quad\left(\varphi_{m}(t)=\theta_{m}(t)+\pi / 2\right)$ are amplitudes of $I_{r}$ and $I_{m}$, the angular frequency difference of the heterodyne light source and a phase to be measured. The active phase shifter adjusts the phase of $I_{r}$, then the phase-shifted refrence signal $I^{\prime} r$ is,

$I_{r}^{\prime}=A \sin (\Delta \omega t+\Phi)$

where $\Phi$ is an adjustable phase in the PLL. At the mixer, the product of $I_{m}$ and $I^{\prime} r$ is obtained as follows;

$$
\begin{aligned}
I^{\prime}{ }_{r} I_{m}= & \frac{1}{2} A B \sin \left(2 \Delta \omega t+\Phi+\theta_{m}(t)\right) \\
& +\frac{1}{2} A B \sin \left(\Phi-\theta_{m}(t)\right)
\end{aligned}
$$

To remove a high-frequency component (the double-beat frequency appearing in Eq. (4)), the product is passed through the LPF, and the output signal after the LPF (= the error signal $\left.\left(O_{r}\right)\right)$ is calculated as

$O_{r}=\frac{1}{2} A B\left(\Phi-\theta_{m}(t)\right) \approx \frac{1}{2} A B\left(\Phi-\theta_{m}(t)\right)$

where we assume $\Phi-\theta_{m}<<1$. The error signal $O_{r}$ should be zero by a negative feedback loop, which utilizes the integrator to create the null condition so that $\Phi$ becomes $\theta_{m}\left(\Phi=\theta_{m}\right)$. When the PLL is locked, the measured $\Phi$ is adjusted to cancel out $\theta_{m}$ and is accumulatively identified by the integrator (a detailed description is given in Section 2.3). Finally, the displacement information can be obtained from the phase shift between the two input heterodyne signals. The detail of operation principle of the phase meter can be seen in [3].

Figures 3(a) and (b) show the measurement results of the displacement measurements of $22 \mathrm{pm}$ and $11 \mathrm{pm}$ mechanical steps, respectively. At the moment, we only confirm $11 \mathrm{pm}$ step using the above implementation.

We would like to express our sincere thanks to Chuo Precision Industrial Co., Ltd for the use of the equipment.

\section{References}

[1] R. Saathof, G. Schutten, J. Spronck, R. Munnig Schmidt, Precis. Eng., 41, (2015) 102-110, https://dx.doi.org/10.1016/j.precisioneng.2015.03.004

[2] F. C. Demarest, Meas. Sci. Technol. 9 (1998) 10241030, https://doi.org/10.1088/0957-0233/9/7/003

[3] T. D. Nguyen, Q. A. Duong, M. Higuchi, T. T. Vu, D. Wei, M. Aketagawa, Sens Actuator A, 304, (2020), 111880. https://doi.org/10.1016/j.sna.2020.111880 